\title{
HACIA UNA REVISIÓN EPISTÉMICA DE LA PRODUCCIÓN CIENTÍFICO-TECNOLÓGICA ENTORNO A PROCESOS INCLUSIVOS DE TRANSFORMACIÓN SOCIAL
}

\section{Paula Peyloubet}

Dra. Arquitecta. Magíster en Desarrollo Urbano. Investigadora adjunta del CONICET (Consejo Nacional de Investigaciones Científicas y Técnicas). Investigadora de CEA (Centro de Estudios Avanzados). Profesora de grado y posgrado de la Facultad de Arquitectura, Urbanismo y Diseño de la Universidad Nacional de Córdoba.

\section{Mariana Ortecho}

Licenciada en Arte. Becaria de posgrado de CONICET (Consejo Nacional de Investigaciones Científicas y Técnicas) en CEA (Centro de Estudios Avanzados). Desarrollo de Tesis Doctoral en CEA - Doctorado en Estudios Sociales de América Latina. Universidad Nacional de Córdoba.

\section{Valeria Fenoglio}

Arquitecta. Becaria de posgrado de CONICET (Consejo Nacional de Investigaciones Científicas y Técnicas) en CEA (Centro de Estudios Avanzados). Desarrollo de Tesis Doctoral en la Facultad de Arquitectura-Universidad Nacional de Tucumán.

\section{Noelia Cejas}

Licenciada en Comunicación Social. Becaria de posgrado de CONICET (Consejo Nacional de Investigaciones Científicas y Técnicas) en CEVE (Centro Experimental de la Vivienda Económica). Desarrollo de Tesis Doctoral en CEA - Doctorado en Estudios Sociales de América Latina. Universidad Nacional de Córdoba.

\section{Institución CEA-CONICET}

Centro de Estudios Avanzados. Unidad Ejecutora de CONICET Espacio multidisciplinario orientado a la investigación y desarrollo en el ámbito de las Ciencias Sociales y Humanidades. Con sede en la Ciudad de Córdoba-Argentina. www.cea-conicet.gov.ar

\section{Institución CEVE-CONICET}

Centro Experimental de la Vivienda Económica. Unidad Ejecutora de CONICET. Con sede en la Ciudad de Córdoba, cuenta con más de 40 años de experiencia en desarrollo de tecnologías constructivas y de gestión abordando la problemática de hábitat desde una visión integral. www.ceve.org.ar

ISNN I666-6I86. Volumen II N II (Octubre 20 II) Pp. 059-079 - Recibido: I5-03-II; Aprobado: I9-08-II 


\title{
$\underline{\text { Resumen }}$
}

El presente artículo plantea algunos cuestionamientos respecto de la base cognitiva con la que el Estado, y su sector de Ciencia y Tecnología, produce abordajes a la problemática habitacional, marcados fuertemente por el determinismo tecnológico. La crítica a esta modalidad no es una novedad en el sector para el cual se escribe, mas la revisión del modelo epistémico a través del cual se piensa el problema y los consecuentes abordajes se consideran aún insuficientes. Por ello se plantean, mediante una mirada transdisciplinar, los fundamentos y rasgos de una epistemología participativa que persigue resultados socialmente inclusivos.

\section{Palabras clave}

Modelo alternativo de producción de conocimiento, producción social de hábitat, tecnología social.

\begin{abstract}
This paper examines the cognitive basis from which the State, and specifically its Science and Technology sector, approaches Social Housing problems restricting its perspective to a technologically deterministic point of view. The critical review of this form of knowledge production might not be taken as innovative by the sector to which this article is directed, but the revision of the epistemic matrix which sustains the construction of the problem itself has not been sufficiently considered. Therefore the authors of this paper, using an interdisciplinary approach, aim to reflect on the foundations and characteristics of a participatory epistemology which tends to produce inclusive outcomes of research.
\end{abstract}

\section{$\underline{\text { Keywords }}$}

Alternative Model of knowledge Production, Social Production of Habitat, Social Technology. 
Hacia una revisión epistémica de la producción científico-tecnológica en torno a procesos inclusivos de transformación social

\section{INTRODUCCIÓN}

La problemática del hábitat en Argentina es una clara manifestación de las tendencias mundiales, caracterizadas por el proceso de globalización de los mercados, la política de ajuste estructural y las políticas sociales focalizadas. Las consecuencias de dichas tendencias se reflejan en los procesos de producción del hábitat, y con mayor profundidad en el campo popular, con una intensa precarización de las condiciones de vida, producida por una profunda crisis socioeconómica de una buena parte de la población.

El crecimiento y la permanencia de dicha problemática ponen en evidencia la ineficacia de los actuales modelos de intervención para generar estrategias de transformación social. En este sentido, el presente trabajo tiene como objetivo reflexionar y cuestionar la base cognitiva con la que el Estado por un lado y el sector de Ciencia y Tecnología por otro intentan resolver la cuestión.

Por un lado, las estrategias utilizadas por el Estado para abordar el problema de hábitat suelen construirse a partir del concepto de déficit, restringiendo la solución a viviendaartefacto e infraestructura-servicio, adoptando una política habitacional de tipo asistencialista y productora de obra pública. Por otro lado, desde CyT, se parte de construcciones problemáticas similares, en las que la carencia de vivienda es entendida como el elemento central. Las soluciones, entonces, se asocian con la necesidad de encontrar una respuesta tecnológica "apropiada", por lo que se producen artefactos, adhiriendo al concepto de transferencia tecnológica, en los que se interviene con tecnologías "stock" delineadas por una elite académica.

Por lo tanto, abordar la problemática del hábitat desde un enfoque centrado en las carencias constituye una visión reduccionista que se encuentra instalada en la construcción problemasolución tanto del Estado como del sector de CyT. En esta reducción epistemológica se recorta, con evidencia, el máximo potencial de resolución del problema, que es el saber de los actores protagonistas, el cual proviene de sus prácticas y experiencias.

En el presente trabajo, la noción de hábitat ${ }^{1}$ se comprende como un elemento complejo, sistémico y democrático. Desde esta perspectiva resulta relevante poner en discusión la relación Hábitat, CyT, Sociedad y las producciones que surgen de ella. En este sentido, se intenta reflexionar sobre la actual base cognitiva con la que se construye esta problemática. 1-Hábitat se entiendecomple-
jo por la múltiple convergen-
cia de campos disciplinares
y abordajes; sistémico por
engendrar un red sinérgica
de elementos constitutivos
que poseen dependencia mu-
tua y afectación colectiva.
Por último, democrático,
por entenderse plenamente
participativo, superando las
instancias de información,
para involucrarse en instan- 
Paula Peyloubet, Mariana Ortecho, Valeria Fenoglio, Noelia Cejas $\overline{\text { 2- La diferenciación entre }}$ 'natural'y 'social' no se usa aquí de modo disyuntivo excluyente. Solo se menciona de modo disgregado a los fines de dar a entender, mediante la representación tradicional, que se quiere aludir a todo aquello que se manifiesta a la percepción.
El artículo se estructura en cuatro partes. La primera gira en torno a cuestionar los abordajes epistémicos tradicionales que suponen valores e intereses implícitos en la producción de un conocimiento que se caracteriza por sostener el paradigma ofertista tecnológico, las transferencias de base lineal, la pretendida neutralidad científica, la emulación de modelos exógenos con escaso o nulo valor local y la tecnología manejada como caja negra. A partir de dicha situación, la segunda parte del trabajo intenta aportar enfoques vinculados con la socialización de los conocimientos, perspectivas transdisciplinares y tecnología social, considerándose este un abordaje cognitivo alternativo. La tercera parte presenta una experiencia, base empírica del artículo, en la que se manifiesta un recorrido desde enfoques epistémicos tradicionales hacia enfoques epistémicos alternativos. Por último, se exponen reflexiones finales.

\section{ENFOQUES EPISTÉMICOS TRADICIONALES: LA CRÍTICA}

$\underline{\text { Sobre la naturaleza de lo científico y lo tecnológico }}$

En distintos y recurrentes intentos por definir la naturaleza de sus identidades, la ciencia tanto como la tecnología han desarrollado definiciones sobre sus propios objetivos, metodologías y resultados, intentando encontrar rasgos diferenciales intrínsecos a uno y otro tipo de actividad.

Quizás resulte prudente reseñar, de modo sucinto, que ha sido la búsqueda de la verdad, entendida como la dilucidación de argumentos explicativos de todo fenómeno natural o social ${ }^{2}$, el camino delineado por el quehacer científico, al menos en el recorrido realizado a lo largo de la tradición occidental de producción de conocimiento. La tecnología, por otra parte y de modo complementario, habría sido entendida entonces como la aplicación o el empleo de estos desarrollos científicos al diseño e implementación de procesos y productos específicos destinados a generar una transformación en el mundo "real", o dicho de modo más preciso: "factual".

A los fines de este trabajo entonces, se considera importante explicitar posicionamientos respecto de las significaciones atribuidas a una y otra expresión. En primer término, y sin entrar en disquisiciones ontológicas, se reconoce a la producción científica como aquella orientada a generar representaciones lógicas y abstractas de la porción manifiesta del mundo circundante e interior. En segundo término, se reconoce a la producción tecnológica como la mediadora del asentamiento del hombre en la realidad, suponiendo transformaciones tanto tangibles como intangibles del mundo material (QueRALTó, 1997). De allí, se asume a la ciencia como vinculada con la generación de teoría y a la tecnología como la aplicación práctica que emplea estos desarrollos formales o conceptuales. 
Hacia una revisión epistémica de la producción científico-tecnológica en torno a procesos inclusivos de transformación social
Ahora bien, aun a riesgo de exponer consideraciones ya bien maduradas en el campo de algunas áreas de conocimiento, y que puedan por tanto aparecer como señalamientos ingenuos, se prefiere aclarar aquí que al referir a "aplicaciones tecnológicas" no se alude de modo exclusivo a artefactos materiales, sino que se incluye en esta nominación a procesos de gestión u organización social que puedan utilizar elaboraciones provenientes de ramas de la ciencia como la antropología, la lingüística o la semiótica, entre otras, como lo presupone LATOUR (2005) en su perspectiva teórica de Actor-Red, reeditada en los ensambles sociales de la sociología de las asociaciones.

Valga decir entonces que todo proceso de producción habitacional incluye diversas aplicaciones tecnológicas. Atender solo el aspecto material, manifestado en el nivel de la vivienda y la infraestructura, implica restringir la mirada a lo perceptible por los sentidos, perdiendo la posibilidad de considerar todas aquellas otras aplicaciones, igualmente tecnológicas, que aun sin poder ser percibidas por dichos sentidos constituyen aspectos centrales y condicionantes de estos procesos, tales como las articulaciones entre actores, las vinculaciones y las controversias, las producciones de sentido sobre el hacer colectivo, el funcionamiento de grupos, la generación de confianzas y los consensos atribuibles a democráticas decisiones.

Más allá de las aclaraciones pertinentes para el campo del hábitat, es importante comprender el modo en que la Ciencia y la Tecnología han logrado establecer dominios diferenciados, pero sinérgicos, relativamente consensuados en el interior de las ciencias sociales y naturales ${ }^{3}$ (LANDER, 1992). Este razonamiento pretende señalar que, si bien es importante situar posicionamientos respecto de las acepciones atribuidas a una y otra expresión, es asimismo relevante recordar que desde determinado punto de vista estos recortes sémicos solo ocultan algunas de las vinculaciones esenciales que sostienen, contiguas y asociadas, estas dos maneras de generar conocimiento teórico y práctico. Este punto de vista esencialista de los procesos tecnológicos invita a reflexionar sobre los patrones de producción cognitiva, vinculados con diversas prácticas culturales e inseparables de los hábitos sociales que las sustentan.

\subsection{Sobre las herencias positivistas y las tendencias materialistas}

Las consideraciones denominadas hoy "tradicionales" pueden rastrearse en los pilares de la ciencia moderna europea, o dicho de un modo más preciso y justo: "eurocéntrica". Las primeras grietas positivistas, nacidas probablemente en alguna vertiente del idealismo y el pragmatismo filosófico del siglo XIX, fueron ganando territorio en las arenas de la ciencia occidental hasta constituirse en posturas ontológico-epistemológicas de una contundencia hasta hoy irrebatible.
3-Edgardo Lander (1992:14) señala certeramente que 'ciencia' y 'tecnología' se dispusieron en la cultura occidental como 'normativas' orientadas al control eficiente de la naturaleza y la sociedad separadas de todo valor ético, moral o politico que no tuviera por finalidad el control de la 'realidad'. 
Paula Peyloubet, Mariana Ortecho, Valeria Fenoglio, Noelia Cejas
4- Es interesante incluso notar que a pesar de que el uso de un término como 'hábitat' ampliada respecto de los componentes culturales, persiste fuertemente la mirada que restringe por una parte esta noción a 'vivienda', como conjunto de artefactos materiales y por otra parte a organización social, en tanto dimensión vinculada con la capacidad de gestión.

5-Edgardo Lander (2005) ha explicado con detenimiento las afluencias teóricas que nutrieron la formulación de la obra marxista, señalando cómo y por qué puede considerarse eurocentrista. debiera incluir una mirada

El poder de representación de la mente humana se asume actualmente como limitado, y por tanto sus producciones no pueden aspirar a una objetividad impecable que formule enunciados desvinculados de sus condiciones culturales de configuración, generalizables a otros universos diferentes de los que le dieron emergencia. Pero si la realidad solo puede ser percibida de modo parcial, el conocimiento debe entenderse como un conjunto de representaciones en permanente transformación; rasgo que lo vuelve "perfectible" a los ojos del pospositivismo, "localizable" a la mirada de las teorías críticas geopolíticas y "fragmentario" e incluso "errático" para el punto más extremo del posmodernismo.

Cada corriente teórica parece así haber encontrado su lógica argumental para atacar la ingenuidad positivista y encontrar un nuevo bastión desde el cual defender una ciencia que precisamente se erija como algo más que una práctica netamente instrumental. Sin embargo, aun habiendo recorrido estos caminos, muchas de las investigaciones actuales, en el campo de las problemáticas habitacionales, parecen no lograr librarse de una serie de hábitos heredados desde una tradición positivista que, tal como se mencionó anteriormente, privilegia la dimensión material así como su relevamiento cuantitativo al abordar problemáticas vinculadas con el complejo fenómeno de "habitar". 4

Pareciera que el "materialismo" como corriente filosófica y como basamento ontológico del marxismo - considerando a este último como una de las corrientes más fuertes del pensamiento social del último siglo — sería el primer punto del cual muchas nuevas corrientes intentan alejarse. ${ }^{5}$ La idea de que el elemento central, condicionante en el desarrollo de la vida social, sería nada más y nada menos que el sistema de producción y distribución ha contribuido a naturalizar una visión que privilegia la dimensión económica por sobre otros aspectos que organizan el andamiaje cultural civilizatorio occidental, dificultando otras facultades humanas que explican otras formas de conocer y saber.

En este sentido, se explica el privilegio de la razón por sobre otras facultades humanas que han sido relegadas, e incluso negadas, como componentes relevantes para considerar en la producción científica social, aunque su inclusión es inaplazable. La aspiración de objetividad, como pilar epistemológico, está en franca crisis y va siendo desplazada por posturas que intentan poner de manifiesto la dimensión subjetiva de la organización simbólica societal. Por tanto, hoy la ciencia está más cerca de aceptar que ningún sistema de producción delinea relaciones de dominación, sino que, por el contrario, son determinados patrones de relación, en una primera escala interpersonal, los que sostienen y promueven estas disposiciones que a otro nivel estructuran el andamiaje social. 


\subsection{Sobre las relaciones interactorales asimétricas}

Tal como están planteados los abordajes tradicionales de desarrollo científico y tecnológico, se observa la reproducción de ese orden vertical, unidireccional, en las prácticas sociales que se desprenden de ellos. En tanto la base cognitiva de producción de conocimiento permanezca sectorizada, difícilmente se logrará superar el estado de desigualdad social que, aun con las mejores intenciones, no es factible de generar transformaciones dada la constante estructural: las relaciones asimétricas entre los actores implicados en la construcción del problema y de la solución del campo que se aborda.

El modelo que concibe este tipo de relaciones, en el que la definición del escenario problemático y su correspondiente resolución están definidos por los sectores hegemónicos, históricamente legitimados e instituidos, configura en el campo una red de relaciones que inhibe a algunos actores de introducir su propia mirada. Esta configuración asimétrica de las relaciones interactorales hunde sus raíces en dos supuestos, heredados del paradigma positivista, el cual plantea un vínculo que diferencia entre un sujeto cognoscente, el investigador, y un "objeto" por conocer, que muchas veces es, ni más ni menos, que otro sujeto o grupo de sujetos, tan social, histórica y culturalmente condicionado como el investigador (VASILACHIS I., 2007).

Otra de las herencias, también en el plano de lo teórico, que se desprende de la primera es el supuesto de racionalidad científica que propone la distinción entre el conocimiento científico y el conocimiento de sentido común o popular, estableciendo como legítimo al primero en detrimento del segundo (SANTOS B., 2009). La diferenciación de tipos de saber no es intrínsecamente perniciosa, dado que la jerarquización de los conocimientos es una acción inherente a toda práctica social; en tal caso, lo nocivo de esta diferenciación es la categorización superlativa del saber científico, siempre superior al conocimiento popular; ipso facto son puestos en comparación (SANTOS B., 2009).

En un plano empírico social, la institucionalización de este estado de cosas provoca la aceptación e interiorización de esa asignación de roles y jerarquías, que se hace manifiesta en la objetividad de las estructuras sociales que rigen las prácticas y en la subjetividad de las estructuras mentales de quienes se ven sumidos en ellas (TeBeroski, A. 1996). De esta manera, la relación asimétrica se traduce en una relación que acepta la dualidad dominio/ dependencia, la cual, una vez internalizada, puede ser un obstáculo al momento de pensar en nuevas formas, más participativas, respetuosas de saberes diversos, en la resolución de problemáticas que históricamente habían sido acotadas, restringidas, definidas y abordadas por el conocimiento técnico de "los que saben". 
Habiendo señalado esto, cabe reflexionar en torno a las verdaderas posibilidades que proporcionan el actual modelo de desarrollo y su correspondiente base cognitiva a fin de producir una transformación social. ¿Cómo generar inclusión social sin inclusión epistemológica? Es decir, si se pretende generar nuevos abordajes, que permitan el desarrollo de prácticas sociales inclusivas, es esencial la búsqueda de estrategias de producción de conocimiento que garanticen la participación de los grupos sociales involucrados en esto que se busca resolver (SANTOS B, 2009), particularmente para la temática que nos convoca, la búsqueda de soluciones habitacionales debiera contemplar estrategias que tiendan a horizontalizar las relaciones, en dos sentidos: asumir la transdisciplinariedad en la complejidad del campo de la producción social del hábitat $\mathrm{y}$, especialmente, integrar en la definición del abordaje a los protagonistas de la situación que se intenta resolver, con sus saberes y sus experiencias, no solo desde sus carencias.

\subsection{Sobre el determinismo tecnológico}

En la actualidad los procesos de construcción de conocimiento, en las instituciones de CyT públicas, son desarrollados en la mayoría de los casos por actores participantes sin reconocer lo que la neutralidad científica y el determinismo tecnológico presuponen (Dagnino, 2008).

Cuando hacemos referencia a una tecnología y por consiguiente tendemos a definirla, suele representarse imaginariamente la idea de un artefacto, en tanto aparato aislado medido por su grado de eficiencia. Es decir, existe una tendencia a comprender la tecnología de un modo artefactual y de separar dichos productos tecnológicos de su contexto social, político y cultural. Este intento de separar la tecnología de la sociedad nos coloca en determinismos no deseados. Desde una visión crítica, FEENBERG (1991) argumenta que el determinismo tecnológico se apoya en el supuesto de que las tecnologías tienen una lógica funcional autónoma que puede ser explicada sin referencia a la sociedad. Siguiendo también una línea crítica, DAGNINo (2007) señala que la visión de la tecnología actual es determinista, siendo además autónoma y neutral, destacando el hecho de que la Ciencia y la Tecnología son condicionadas por valores desde su producción y que por lo tanto es un equívoco creer que, mediante determinados posicionamientos éticos, esa tecnología va a ser utilizada para el "bien" o el "mal". Esta condición confiere, por lo tanto, a los desarrollos de tecno-ciencia características específicas que reproducen un único modelo de desarrollo, promovido desde y para países ricos, que finalmente inhiben una verdadera transformación social.

Según Thomas (2009) las tecnologías son construcciones sociales tanto como las sociedades son construcciones tecnológicas. Desde esta perspectiva teórica, enfoque sociotécnico, 
resulta prioritario abordar la problemática habitacional superando y rechazando todo tipo de determinismos para dar lugar a una especie de entramado donde lo tecnológico no determina lo social y lo social no determina lo tecnológico. También resulta relevante generar un cambio radical en la concepción básica de la tecnología, rechazando el determinismo e incorporando nuevos valores que promuevan espacios democráticos en decisiones tecnológicas, como así también procesos inclusivos y solidarios.

Una postura radicalmente opuesta al determinismo tecnológico es el concepto de adecuación sociotécnica (DAGNINO, 2007), que plantea la idea de establecer valores alternativos a priori en la producción de la Tecnología. "La adecuación socio-técnica hace referencia a un proceso que busca promover adecuación de conocimiento cientifico-tecnológico -incorporado en equipamientos, insumos, formas de organización de la producción o inclusive en forma intangible o tácita - no solo a los requisitos y finalidades de carácter técnico y económico, sino al conjunto de aspectos de naturaleza socioeconómica y ambiental vinculados con la participación democrática en el proceso de trabajo, la atención al ambiente, a la salud de los trabajadores y consumidores, y a su capacitación autogestionaria" (DAGNino et ál.; 2004: 52).

En este sentido, abordar la problemática del hábitat desde esta perspectiva teórica implica generar procesos diferenciados en la construcción del problema. El uso de tecnologías sociotécnicamente adecuadas promueve la construcción de conocimiento de naturaleza endógena, reivindicando el conocimiento local y la promoción de procesos vinculados con la participación democrática de todos los actores involucrados en la resolución del problema. Por lo tanto, resulta prioritario reorientar la producción de hábitat hacia el uso de tecnologías que superen las contradicciones y restricciones del abordaje determinista.

\subsection{Sobre el conocimiento neutral}

Este apartado surge de un postulado concreto: el conocimiento no es neutral. Si bien cierta postura epistemológica puede construir para sí criterios de objetividad, estos derivan de juicios ético-políticos arbitrarios, intencionales, preexistentes a la construcción de conocimientos. Surgen de una construcción de la realidad y de un posicionamiento en ella expresando una edificación de pensamientos, saberes articulados, que no pueden ser neutrales (SANTOS, B. 2009). De modo que las visiones hegemónicas positivistas, que luego se traducen en decisiones que condicionan las prácticas sociales, proponen un tipo de desarrollo que involucra a toda la sociedad, pero que no necesariamente se traduce en una mejora para todos sus miembros. 
Pensar que los escenarios de CYT son neutrales es ingenuo, ya que esos escenarios y sus actores también están atravesados por condiciones históricas, políticas, económicas, etcétera, que propician y en algún sentido determinan el avance del conocimiento en un sentido y no en otro. Por ello, resulta interesante reflexionar en torno a los valores puestos explícita o implícitamente en juego. Este gesto implica una doble mirada, tanto de los condicionamientos que existen en el campo CyT tal cual está dado, como también los compromisos que asume o debe asumir en el vasto campo sociohabitacional.

Desde este punto de vista, es imposible pensar que el desarrollo tecnológico para el hábitat se traducirá inexorablemente en bienestar social (DAGNINO 2003). Basta con echar una mirada cualitativa, antes que cuantitativa, sobre los efectos de ese tipo de desarrollo para reconocer que es indispensable plantear nuevos modos de conocer y hacer, que superen las posturas que aún defienden la neutralidad de la ciencia o el determinismo tecnológico, para lo cual es necesario desarrollar una nueva base cognitiva. Los esbozos de pensamiento en torno a ese esfuerzo propuestos por este equipo serán expuestos en el apartado contiguo, pero vale decir aquí que esa nueva lógica de producción de conocimiento debe proponer puentes que unan miradas y saberes diversos, reconociendo la no neutralidad, asumiendo la intersubjetividad e incorporando la pluralidad de cosmovisiones (saberes, idiosincrasias, deseos y necesidades) de los diversos actores que entran en este nuevo juego.

Así, la producción de conocimiento debería contemplar saberes académicos y saberes populares, en una construcción colectiva, tendiente a la inclusión social en el marco de la transformación de modelos cognitivos que propicien la transformación social en el escenario problemático que se pretende abordar. Del mismo modo, es necesario diluir las fronteras disciplinares que limitan esos escenarios complejos, múltiplemente atravesados, con reduccionismos que los hacen "manejables" en la profundidad del campo académico mas no en el campo empírico. Es decir, comenzar por hacer transversal, a todos los campos, los valores sociales podría ser solo un comienzo. La tendencia sería asumir esta estructura con todos los campos disciplinares, la transdisciplina como mirada complementaria, tal cual se expresa en líneas anteriores.

\section{ENFOQUES EPISTÉMICOS ALTERNATIVOS: LA PROPUESTA}

3.1. A partir de miradas integrales transdisciplinares

La complejidad, como noción y rasgo constitutivo de "lo real", ya ha sido amplia y sólidamente fundamentada por reconocidos y numerosos teóricos (Prigogine, 1994; Morin, 2004). Los argumentos más prominentes probablemente puedan encontrarse en la obra 
de Morin y particularmente en sus reflexiones en torno a las posibilidades contemporáneas de construcción de un paradigma epistémico lo suficientemente dinámico y flexible como para escapar de las trampas de la razón que suponen simplificaciones, reducciones y disyunciones, características sobre las que se sostuvieron muchos de los grandes descubrimientos en el campo de las ciencias naturales, y que hoy son repensadas tanto para las ciencias sociales, campo que nos ocupa en este artículo, como para las propias ciencias naturales (STENGERS 2002).

Dentro de las múltiples características reduccionistas del patrón de construcción de conocimiento moderno, una de las más difíciles de superar, probablemente esté asociada con la cuestión de la compartimentación disciplinar que, habiéndose generado en el interior de la institución académica en tanto segmentación de áreas de conocimiento, ha rebasado los límites de este campo para constituirse en una matriz de comprensión y representación de todo aquello que se manifiesta a nuestra percepción. Considerar, por ejemplo, cualquier fenómeno social vinculado con procesos habitacionales puede albergar una serie de miradas que, desde ciertas especialidades, observen con detenimiento aspectos productivos, técnico-constructivos u organizacionales, pero lo que no debiera permitirse pasar por alto es construir un punto de vista que ponga en relación estos elementos en el interior de un marco más amplio que aborde la cuestión simbólico-cultural en la que todos estos "productos" y "procesos" tienen lugar.

Asimismo una mirada con voluntad genuinamente holística encontraría conveniente vincular dos niveles fundamentales de lectura. Se trataría de combinar representaciones explicativas de orden macroestructural con un abordaje cualitativo que desde una mirada micro y local arroje elementos sobre las capacidades de agencia efectiva de los protagonistas de los procesos estudiados. Comprender la dinámica compleja que sostiene el juego de acciones y reacciones entre lo micro y macrosocial es sin duda un desafío teórico y metodológico que recién comienza hoy a asumirse. Contribuir a la emergencia de un nuevo modelo cognitivo y epistémico probablemente requiera la inclusión de estas lógicas circulares y recursivas que encuentren, más allá de las estériles miradas disciplinares, la vinculación entre las macroconfiguraciones políticosociales y aquellos elementos particulares vinculados con la subjetividad de todos aquellos varones y mujeres, que finalmente son quienes "animan" los procesos estudiados.

\subsection{A partir de conocimiento socializado}

En el marco de aportar a la construcción de una base cognitiva diferente, epistemológicamente participativa y metodológicamente dinamizadora de procesos, se considera fun- 
damental la coconstrucción de conocimiento, alimentada por las miradas de los diversos actores que están presentes en el campo problemático que se aborda, que para el caso puede tratarse de la producción social de hábitat, pero no se aplica de manera exclusiva. Se entiende al conocimiento socializado como el final de la ponderación hegemónica del saber científico sobre el saber popular, consuetudinario, ancestral, a razón de que la riqueza que todos estos saberes acumulan pueda aportar a la definición del binomio problemasolución de manera más compleja. En ese sentido, nuestro campo de abordaje brinda todos los elementos para poder desarrollar estrategias de acción tendientes a la integración en una acepción profunda, que invita a hacer partícipes a los miembros de las comunidades con las que se trabaja, afianzando vínculos a fin de desarrollar soluciones habitacionales incluyentes, responsables, respetuosas de la diversidad, con conciencia ambiental e integradoras del saber popular.

La "distribución equitativa del conocimiento" o "democratización del saber" no es suficiente, si por ello se entiende la divulgación del saber científico, pero por sobre todo no es posible en los términos que hoy traza la racionalidad científica (SANTOS, B. 2009). En su lugar, se propone reconocer el valor en otros saberes, de la mirada de otros actores, evitando reproducir en ese reconocimiento relaciones asimétricas vigentes; se trata de dinamizar el acercamiento, la puesta en diálogo de formas de saber no-científicas en igualdad con las científicas. Esto no implica desacreditar el saber científico, sino provocar miradas más complejas resultantes de la asociación entre diversos saberes.

El reto es mucho más que teóricoepistemológico, en todo caso ocupa un lugar preponderante el desarrollo de metodologías que dinamicen estos procesos, que permitan la construcción de puentes entre actores que históricamente han sido acallados en el autismo legitimador de algunos sectores de la comunidad científica, propensa a los modelos positivistas de producción de conocimiento. Así visto, en un plano teórico, se aborda "lo epistemológico" como proceso interactoral, entendido como construcción colectiva de conocimiento, que procura integrar en un plano de "igualdad de oportunidades" a diversos tipos de conocimiento en torno a un problema específico, puestos a dialogar en su configuración, siendo capaces de producir nuevos cuestionamientos provenientes de la experiencia empírica y sus contextos de extrema necesidad. En un plano social, se plantea la inclusión como consigna que está presente desde la génesis del proceso, es decir, no se busca descubrir la panacea que resuelva de una vez y para siempre la desigualdad social, sino de lograr la participación en la búsqueda de soluciones por parte de los actores implicados, en una problemática que presenta cualidades específicas, en un escenario específico y con resultados que serán legítimos en tanto den respuesta en un escenario local. 
Hacia una revisión epistémica de la producción científico-tecnológica en torno a procesos inclusivos de transformación social
Está claro que lo expuesto con este título no es más que un esbozo de lo que se considera relevante de pensar en un esfuerzo que procura la transformación social desde una mirada inclusiva, y seguramente deja por fuera una serie de elementos, justamente por tratarse de un pensamiento embrionario. Si bien las precisiones del modelo cognitivo alternativo aún son tenues, se tiene la certeza de que es necesario introducir transformaciones al modelo actual de producción de conocimiento científico y de desarrollo tecnológico, que tienda puentes hacia la mirada de otros actores, buscando la maximización de aportes a la construcción de "otro mundo posible". 6

\subsection{A partir de tecnología social}

Abordar la problemática del hábitat desde un enfoque centrado en las carencias, utilizando herramientas exógenas de intervención, sin la participación de los usuarios en la resolución y tomas de decisiones, constituye una visión reduccionista en la relación problema-solución. Según Peyloubet (2010) a partir de esta visión se elabora la solución, con un modelo de oferta-demanda que supone una necesidad y un satisfactor, un emisor y un receptor. Este proceso supone una concepción y construcción del conocimiento, materializado a través de una tecnología, por ejemplo una vivienda, que es transferida como solución técnica al problema. En este sentido, el diseño e implementación de resoluciones a la problemática del hábitat son llevados a cabo desde ese paradigma tecnológico que es la transferencia. Esta concepción se encuentra instalada en el abordaje inicial de construcción del problemasolución de las Tecnologías Convencionales ${ }^{7}$ y también en aquellas que surgieron para dar respuestas a los problemas sociales como las Tecnologías Apropiadas ${ }^{8}$, Intermedias, etc.

Este modo de abordar la problemática del hábitat, caracterizado por una transferencia tecnológica, que ignora el conocimiento preexistente y las potencialidades locales, es lo que se intenta transformar desde la perspectiva teórica de las Tecnologías Sociales. Dichas tecnologías concebidas a partir de la adecuación sociotécnica intentan participar activamente en procesos de cambio sociopolítico, socioeconómico y sociocultural, como así sustentar procesos de democratización (THOмAs, 2009).

Por lo tanto, los abordajes en CyT para innovar en hábitat deben reconsiderar la base cognitiva existente fundada en epistemias reduccionistas, cuestionando: el paradigma ofertista tecnológico, las transferencias de base lineal, los desarrollos sin valores en su concepción, la emulación de modelos exógenos de alto valor global y escaso o nulo valor local, la formulación de respuestas mágicas - cajas negras - que segregan grupos relevantes en complejos decisorios. Es posible reorientar esta producción de hábitat, en el sentido que
6- Proclama emblemática del Foro Social Mundial, encuentro anual que se celebra desde el año 2002, en el cual diversas organizaciones de la sociedad civil se declaran "en contra de la hegemonía del capital, la destrucción de nuestras culturas, la degradación la naturaleza y el deterioro de la calidad de vida por las corporaciones transnacionales y las politicas antidemocráticas". Se trata de una mirada que compartimos y tras la cual se alinea este artículo.

7- Tecnologías Convencionales (TC): tecnología de lógica empresarial sojuzgada al modelo de desarrollo vigente de mercado libre y capitalismo, sobre las cuales se asienta, a manera de antitesis, la postura de la Tecnología Social, en oposición y concebida como alternativa (Peyloubet, 2008).

8- "Tecnologías Apropiadas: conjunto de técnicas de producción que utiliza de manera óptima los recursos disponibles de cierta sociedad maximizando así su bienestar". (Dagnino, 1976, p. 86. Tecnología apropiada: ¿una alternativa? Citado en Peyloubet, 2008.) 
Paula Peyloubet, Mariana Ortecho, Valeria Fenoglio, Noelia Cejas
9- Se entiende por Tecnología Social, aun siendo un concepto en construcción, a "los productos, técnicas y/o metodologías replicables desarrolladas en la interacción con la comunidad y que representan efectivas soluciones de transformación social" (Dagnino, 2004:33).

10- "Modelo de gestión intersectorial para la formalización de villas, a partir de la revisión de normativa urbana, marco jurídico-político, y la innovación tecnológica de los recursos. Caso Villa la Tela".

11-El equipo de investigación está dirigido por la Dra. Paula Peyloubet y son integrantes actuales las siguientes investigadoras: Lic. Mariana Ortecho, Abogada Florencia Pasquale, Arq. Ludmila Garbellotto y Lic. Laura Barrionuevo. se viene diciendo, utilizando como herramienta la Tecnología Social ${ }^{9}$, entendida también como una manera pública de acceder a bienes y servicios a partir de la producción de bienes comunes.

Partiendo de la necesidad de generar procesos habitacionales inclusivos, consideramos que abordar la problemática social del hábitat desde esta perspectiva teórica contribuye a generar un enfoque alternativo para el diseño e implementación de estos procesos habitacionales a partir del uso de tecnologías adecuadas sociotécnicamente.

\section{BASE EMPÍRICA DEL TRABAJO: CASO "VILLA LA TELA", CIUDAD DE CÓRDOBA}

\subsection{Presentación del caso}

El caso, que se presenta en este artículo, trata acerca de una experiencia que se está realizando en el marco de un proyecto de investigación, financiado por el Ministerio de Ciencia y Tecnología de la provincia de Córdoba ${ }^{10}$ desde el año 2009. Algunas de las autoras son parte del equipo de investigación. ${ }^{11} \mathrm{La}$ investigación tiene lugar en Córdoba, precisamente en Villa La Tela, asentamiento popular ubicado en el oeste de la ciudad, sobre la ruta provincial $\mathrm{N}^{\circ} 20$, principal arteria que llega hasta el turístico Valle de Punilla de la provincia.

Villa La Tela es un barrio de aproximadamente 40 años de antigüedad, cuyas características son muy particulares en tanto reúne población pobre heterogénea, familias antiguas, asentadas hace décadas, y familias nuevas de renovación espontánea, como así también inmigrantes de otras regiones del país y de países limítrofes. Se caracteriza por ser una villa muy dinámica en cuanto a su movilidad interna, por cambios de un lote a otro por parte de las familias, como por su movilidad externa, ingresos y egresos de familias al barrio de manera continua. Su extensión es de aproximadamente tres manzanas este-oeste, por diez manzanas norte-sur.

Se encuentra en el límite de una zona militar y su localización fue, y es, blanco de especulaciones tanto urbanas, por la traza de la avenida de circunvalación que nunca se dio, como especulaciones inmobiliarias, por su potencial territorial dentro de la mancha urbana que aún no se ha materializado. 


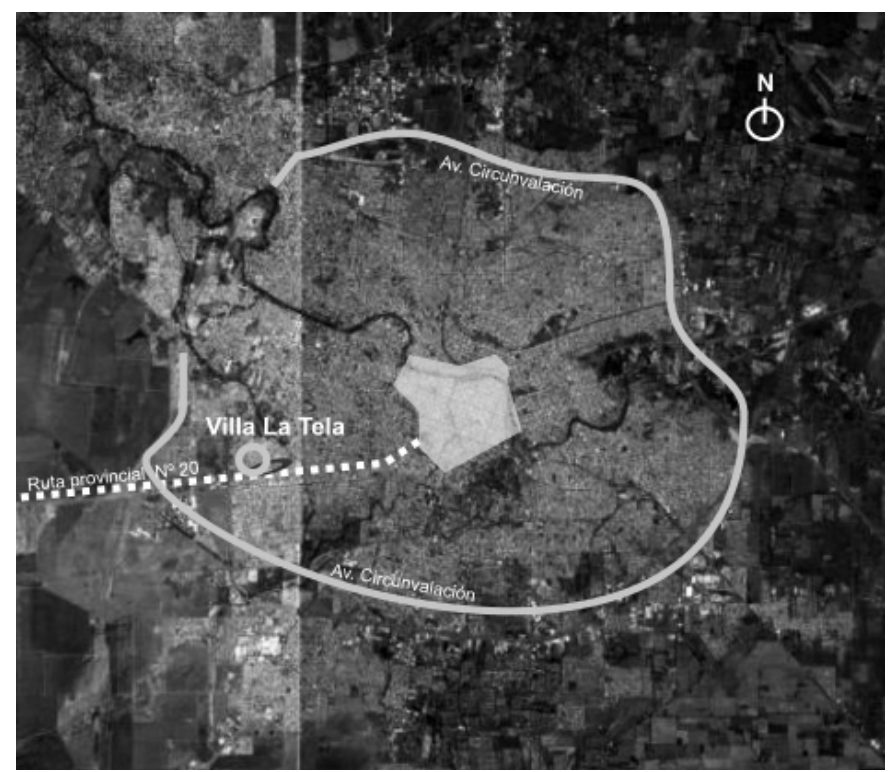

Ubicación geográfica de Villa La Tela.

Fuente: elaboración propia

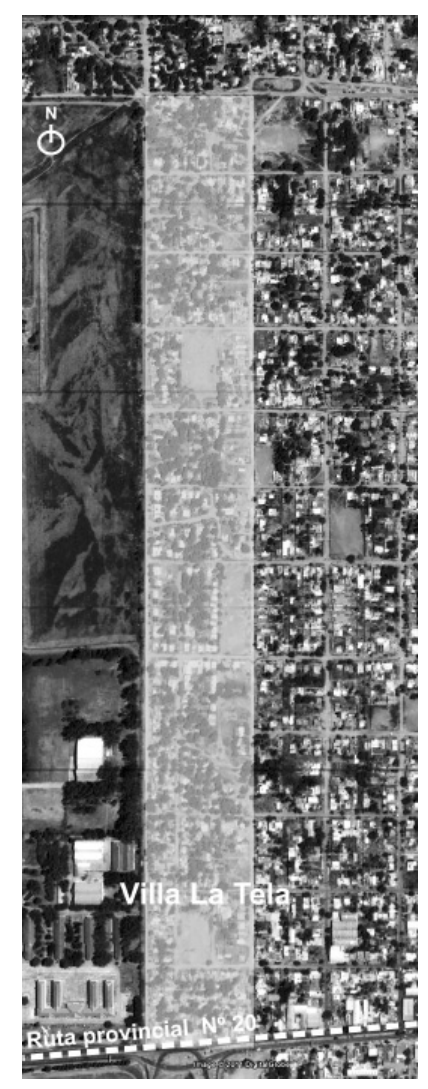

4.2. Categorías "sobre" las cuales producir cambios

Se presenta este proyecto de investigación como caso ejemplificador de un recorrido que transita desde un típico enfoque epistémico tradicional, al momento en que fue formulado en el inicio de la investigación, hacia un enfoque epistémico alternativo, momento actual que acumula unos $2 / 3$ de la investigación planificada. 


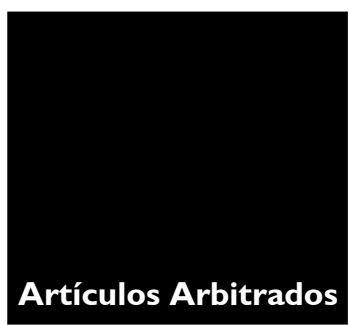

Paula Peyloubet, Mariana Ortecho, Valeria Fenoglio, Noelia Cejas

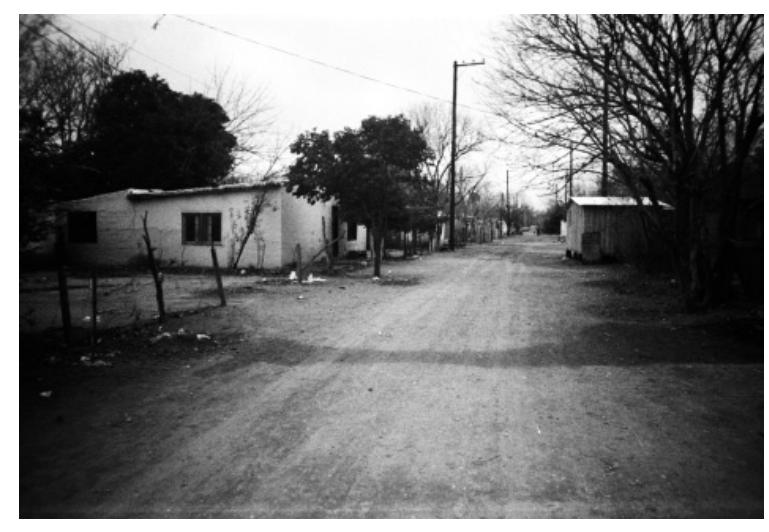

Villa La Tela

Fuente: Peyloubet, 2006. Tesis Doctoral.

"Hábitat Popular Progresivo"

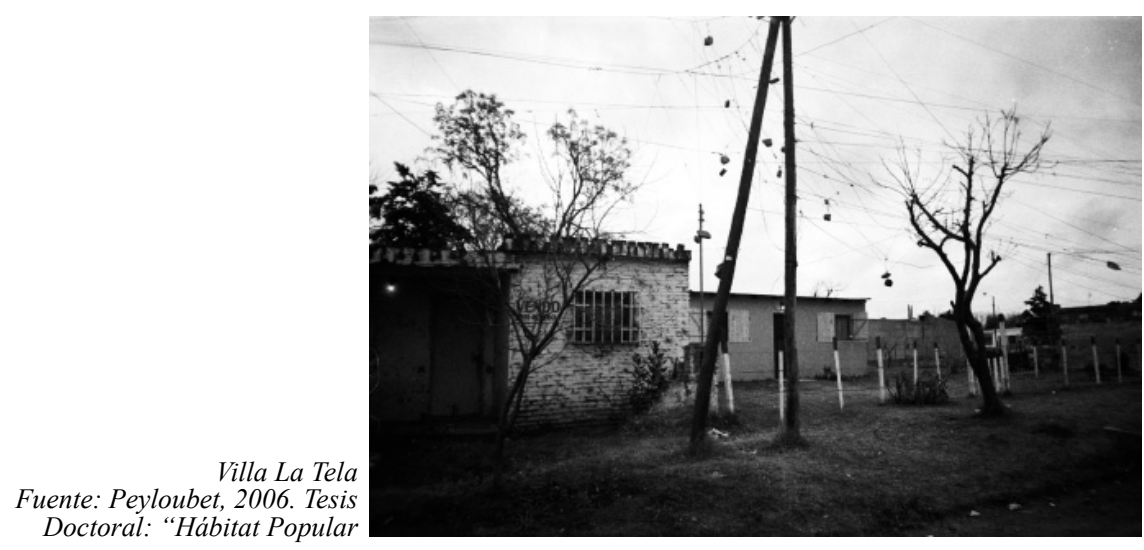

El equipo de investigación inicia el proyecto adhiriendo a ciertos presupuestos técnicos consabidos, resultados de diagnósticos realizados por un sector académico, CyT, y político, datos municipales del plan director para la ciudad, que caracterizan las zonas pobres, en términos de carencias materiales, y que permite reconocer áreas vulnerables donde es previsible intervenir con mejoramientos barriales, vinculados tanto con viviendas como con la realización de infraestructura básica, emulando el modelo de planificación brasileño 
representado por sus estudios urbanos y sus detecciones de las famosas áreas especiales: ZEIS. Por otro lado, la noción del equipo de investigación respecto de propiedad adscribía, en ese momento inicial, a aquella propuesta por el derecho civil, que preserva la propiedad individual por sobre cualquier otra, por lo que la regularización dominial es convertida en una pieza clave de los presupuestos técnicos iniciales del recorrido investigativo.

En este sentido, el proyecto comienza su andar intentando describir de manera cuantitativa, herencia del saber positivista y de tendencia materialista, la situación actual de la villa, en términos de condiciones habitacionales con un fuerte sesgo técnico-constructivo. Para ello, se implementó una ficha de relevamiento familiar, que confirme el presupuesto técnico, legitimando el saber científico y tecnológico apriorístico respecto de los asentamientos pobres en Córdoba, por lo que se decidió tomar una muestra representativa de la villa que arrojara estadísticamente los valores de verdad material, a partir de una fórmula matemática con desviación del 5\%, valor aceptable para la condición de verdad en el criterio científico.

Los objetivos del proyecto de investigación en Villa la Tela ponderaron, en su primera formulación, un abordaje determinista tecnológico, abogando por el desarrollo de mejoras habitacionales así como la regularización dominial. Sin lugar a dudas, esa instancia del proyecto ilustra un abordaje cognitivo basado en criterios de verdad científica y tecnológica, construidos desde un único y poderoso sector: la elite académica y universitaria, situación que evidencia las relaciones asimétricas y las construcciones de conocimiento con criterios de validación sectorial, que nada tienen que ver con enfoques pluriversales, en los que los saberes diversos se reivindican y los criterios de validez de la verdad se representan a partir de una construcción colectiva de conocimientos, tanto académicoscodificados, como populares-tácitos.

\subsection{Categorías "a partir" de las cuales generar el cambio}

$\mathrm{Al}$ iniciar las actividades de campo en Villa la Tela, desde el posicionamiento antes descrito, se empezaron a reconocer las limitaciones del enfoque, lo cual impulsó una serie de cambios. Es allí mismo donde el posicionamiento epistémico tradicionalista comienza a reconvertirse en un enfoque diferente, en el cual el primer cambio consistió en eliminar la muestra representativa con la intención de reconocer la totalidad de la comunidad que vive allí, ante la elocuente heterogeneidad del escenario y sus protagonistas. La oportunidad de rastreo y reconocimiento, a partir de la ficha-encuesta, permite al equipo generar otra premisa investigativa y comienza a reconocer las particularidades frente a las universalidades, iniciando con ello un camino de construcción de confianzas mutuas entre los vecinos 


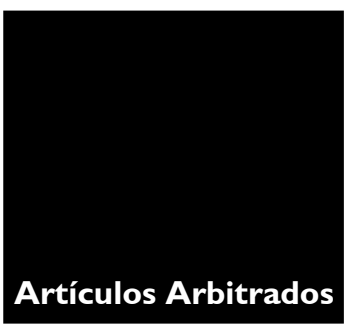

\section{Paula Peyloubet, Mariana Ortecho, Valeria Fenoglio, Noelia Cejas}

de la villa y el equipo de investigación. El valor de la encuesta cambia radicalmente y se convierte en la excusa perfecta para producir el acercamiento mutuo. Los datos de la encuesta dejan de tener la importancia que antes representaban y se la proclama como herramienta de vinculación, vaciada su intención de reconocimiento cuantitativo.

La situación problemática inicial, de mejoramiento barrial y regularización dominial, comienza a calificarse como una obviedad excesiva, y paralelamente a esto aparecen en el territorio, producciones de sentido subyacentes de la propia comunidad barrial que evidencian otros y nuevos relatos de verdad y necesidad. El problema inicial comienza a deconstruirse para dar lugar a una nueva noción de problema, surgida de la apelación de los propios vecinos, en compañía del equipo de investigación.

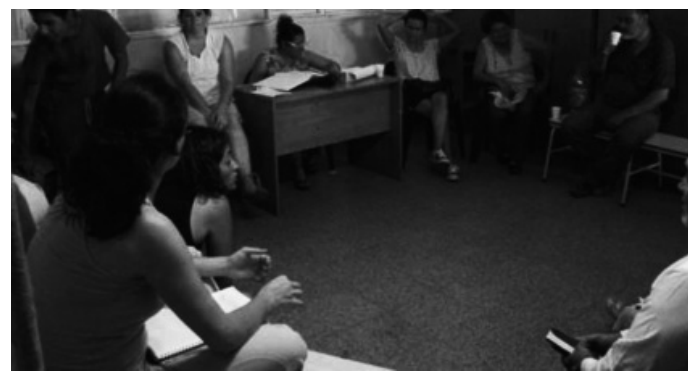

Reuniones con los vecinos

Fuente: PID 2008 MINCYT-CBA

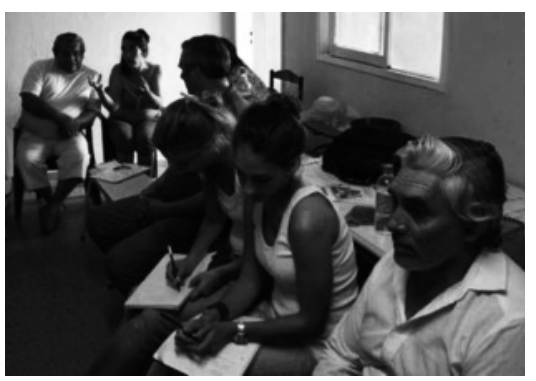

Reuniones con los vecinos

Fuente: PID 2008 MINCYT-CBA

Aparecen en escena nuevos actores sociales, como referentes locales, generando una expresión de necesidad compleja que manifiesta una problemática en la que se superponen condiciones clásicas de carencia material con condiciones poco consideradas de potencialidad humana. Las prácticas y saberes construidos a partir de experiencias endógenas, colectivas e individuales, hacen presuponer producciones de solución diferentes de las imaginadas en el inicio del proyecto y se vuelven ineludibles en el marco de la indagación iniciada.

Actualmente las condiciones de investigación son muy diferentes de las del momento inicial. El lugar donde el equipo está posicionado hoy constituye un importante esfuerzo para contribuir a la construcción de abordajes epistémicos alternativos. Se reconoce esencialmente la mirada del otro, como productora de conocimiento. Se plantea un proceso de construcción colectiva en el que las referencias del problema y su consecuente solución 
son producidas socialmente entre los diversos sectores: coconstrucción. La condición de carencia material es asumida desde una perspectiva de potencialidades en la cual las autonomías y las construcciones endógenas son los principales motores de marcha para cualquier transformación social. Las cajas negras se han abierto y el sentido de la verdad está comenzando a democratizarse.

El principal desafío del proyecto de investigación en este tercio final es encontrar metodologías que dinamicen procesos de construcción colectiva, en los que la producción de verdad se legitime en el saber diverso, generando puentes entre actores cuyos conocimientos operen simultáneamente en la construcción del problema-solución, evitando actividades de autolegitimación por parte del equipo de investigación.

\section{REFLEXIONES FINALES}

El presente artículo ha pretendido reflexionar sobre ciertas cuestiones inherentes a la necesidad de repensar los actuales abordajes, desde una perspectiva general de CyT, en el marco de la cual se ubican las indagaciones en torno a la problemática de hábitat. La base cognitiva actual con que se comprende e intenta resolver el problema referido a hábitat, entendido desde un enfoque complejo en el que intervienen múltiples aspectos interrelacionados sinérgicamente, requiere necesariamente de una revisión epistémica.

Durante los últimos 50 años el concepto relacionado con el hábitat ha evolucionado desde una definición material, en los inicios representada por "necesidad de vivienda", pasando por una visión más compleja que sumó aspectos económicos, sociales, culturales, ambientales, etc., recalando en la actualidad en interesantes acercamientos a perspectivas políticas de derechos y reivindicaciones humanas. La clave para comprender la posición argumental de este artículo es reconocer que, en el campo del hábitat, los postulados conceptuales que definen el objeto de estudio han evolucionado dinámicamente en el tiempo, tal como se expresa en el párrafo anterior, mientras que los abordajes epistemológicos han presentado transformaciones más lentas.

Se asume en este trabajo una posición crítica respecto de la mirada convencional del conocimiento construido para resolver problemas de hábitat, ya que suele reducirse a aspectos tecnológicos, expresados en el déficit material o productivo, sin considerar los procesos en su complejidad, los cuales solo pueden ser comprendidos en términos transdisciplinares desde su génesis. Tampoco se ha renovado la tradición elitista que confiere a la academia 
el rol de experto para solucionar los problemas. En esta condición, la naturaleza de las resoluciones en el campo del hábitat tiende a ignorar saberes no académicos, tácitos y no codificados, perdiendo la oportunidad de poner en juego la creatividad resolutiva de la sabiduría popular. En este sentido, el trabajo pretendió aportar sobre la cuestión de la problematización de la situación. Tal como se comprenda el problema, que trasciende al hábitat, es como se construirán las soluciones. Lo que se está diciendo en estos dos últimos párrafos busca rescatar la necesidad de repensar colectivamente el problema en su compleja manifestación, como expresión integrada de la sociedad, reivindicando las voces en su diversidad. Todos construimos el problema, luego todos solucionamos el problema.

Los valores que subyacen en las investigaciones referidas a hábitat suelen relacionarse con componentes deterministas y neutrales, fuertemente condicionados por la visión positivista, históricamente construidos sobre argumentos atentos a la dimensión material y orientados a la búsqueda de "la verdad". La posición que nos reúne a lo largo del artículo afianza la idea de que la CyT, asumidas como instrumentos del hacer humano, colaboran con el reconocimiento de la realidad y procuran aportar conocimientos a un colectivo de saberes, que se entienden múltiples y diversos. Para ello a lo largo del trabajo se posiciona y se adhiere a una teoría crítica que reconoce y reclama los límites de las soluciones a partir de los enfoques de base epistemológica simplificadora, disyuntora y reduccionista.

Los postulados que pueden arrojar luz sobre la base cognitiva de los abordajes investigativos, que sumerge a cambios a CyT, podrían ser: a) que la construcción del problema define la posibilidad de la solución; b) que los saberes mixtos reivindican los acervos culturales y el stock de conocimientos, codificados o tácitos, de que se dispone para la solución de los problemas; c) que las construcciones colectivas son potencialmente más posibilitantes que las construcciones sectorizadas y elitistas, constituyendo una coconstrucción democrática de dominio público; d) que las soluciones no son universales ni constituyen verdades atemporales, sino producciones ancladas en tiempos y espacios específicos; e) que la base cognitiva es el instrumento disponible con que se piensan los problemas y se construyen las soluciones, por lo que debe estar en continua revisión aceptando que pueden mutar en función de las ideologías imperantes, por lo cual constituye un desafío repensarla sobre la base de modelos de desarrollo inclusores. 
Hacia una revisión epistémica de la producción científico-tecnológica en torno a procesos inclusivos de transformación social

\section{BIBLIOGRAFÍA}

DAGNINO, Renato (2003). "Ciencia y tecnología para pocos”. Revista Eletronica Divulgon. Diciembre. Disponible en http://www.divulgon.com.ar/diciembre03/cys-dic03.html. DAGNINO, Renato (2004) A tecnología social e seus desafios. Tecnología Social. Uma estrategia para o desenvolvimento. CIP Brasil. Rio de Janeiro.

DAGNINO, Renato (2007). "Empezando por la extensión universitaria". Ponencia publicada en Ciencia y Tecnología para el Hábitat Popular. Desarrollo tecnológico alternativo para la producción social del hábitat". Córdoba, Argentina.

DAGNINO, Renato (2008) Neutralidad da ciencia e determinismo tecnológico. Editora UNICAMP. Campina Grande. Brasil.

FEENBERG, Andrew (1991) Critical theory of technology. New York, Oxford. University Press.

LANDER, Edgardo (1992) La ciencia y la tecnología como asuntos políticos. Editorial Nueva Sociedad. Caracas, Venezuela.

LANDER, Edgardo (2005). "Marxismo, eurocentrismo y colonialismo", en La teoría marxista hoy. Atilio Borón (comp.). CLACSO. Buenos Aires, Argentina.

LATOUR, Bruno (2008) Reensamblar lo social. Una introducción a la teoría del actor red. Ed. Manatial. Buenos Aires, Argentina.

MORIN, Edgar (2004) El paradigma de la complejidad. Introducción al pensamiento complejo. Gedisa. Barcelona, España.

QUERALTÓ, Ramón. (1993) Mundo, Tecnología y Razón en el fin de la modernidad. ¿Hacia el hombre more técnico? Ed. PPU. SA. Barcelona, España.

PEYLOUBET, Paula et ál. (2010). "Modelo cognoscente que re-signifique el binomio problema-solución. Perspectiva perceptiva y metodológica”. Ponencia presentada en Esocite 2010. VIII Jornadas Latinoamericanas de Estudios Sociales de la Ciencia y Tecnología. Ciencia y Tecnología para la inclusión Social. Buenos Aires, Argentina.

PRIGOGINE, I. y STENGERS, I. (1983) La nueva alianza. Alianza Editorial. Madrid, España.

SANTOS, Boaventura de Sousa (2009) Una epistemología del sur: la reivindicación del conocimiento y la emancipación social. Siglo XXI. CLACSO, México.

STENGERS, Isabelle (2002) Penser avec Whitehead: une libre et sauvage céation de concepts. Gallimard. Paris, Francia.TEBEROSKI, Ana (1996). "Co-constructivismo y relaciones asimétricas”. En: Anuario de Psicología, pp. 83-87. Universidad de Barcelona, España.

THOMAS, Hernán (2009). "De las tecnologías apropiadas a las tecnologías sociales. Conceptos / Estrategias / Diseños / Acciones". Ponencia presentada al IV Seminario Iberoamericano de Ciencia y Tecnología organizado por el Centro Experimental de la Vivienda Económica del 23 al 25 de septiembre de 2009 en la Ciudad de Córdoba, Argentina.

VASILACHIS de Gialdino, Irene (dir.). (2007) Estrategias de Investigación Cualitativa. Editorial Gedisa. Buenos Aires, Argentina. 
